\title{
INFLUENCE OF OCCLUSAL THICKNESS OF CUSTOM-MADE MOUTHGUARDS ON VENTILATORY PARAMETERS, RATE OF PERCEIVED EXERTION, AND PEAK VELOCITY ATTAINED DURING AN INCREMENTAL TEST
}

\author{
João Antônio Gesser Raimundo', Karina Maria Pires ${ }^{2}$, Ana Clara Loch Padilha ${ }^{3}$, \\ Rafael Penteado $^{1}$, Felipe Domingos Lisbôa ${ }^{1}$, Dayane Machado Ribeiro ${ }^{2}$, and Fabrizio Caputo \\ ${ }^{1}$ Human Performance Research Group, Center for Health and Sport Science, \\ Santa Catarina State University, Florianópolis, Brazil \\ ${ }^{2}$ Department of Dentistry, Federal University of Santa Catarina, Florianópolis, Brazil \\ ${ }^{3}$ Group of Studies and Research on Interdisciplinarity, Education and Health, \\ Federal University of Santa Catarina, Florianópolis, Brazil
}

Original scientific paper

DOI: $10.26582 / \mathrm{k} .51 .2 .5$

\begin{abstract}
:
Custom-made mouthguards are used to prevent orofacial injuries arising from falls and knocks. It has been observed that thicker custom-made mouthguards transmit less force to the mouth due to their higher energy absorption capacity. However, it is believed that thicker custom-made mouthguards can alter ventilation during exercise because of a higher resistance to or restriction of oral airflow. The purpose of this study was to investigate if a thicker custom-made mouthguard (occlusal thickness of 5-mm; 5MG) altered ventilatory parameters, rate of perceived exertion (RPE), and peak velocity during an incremental test relative to a thinner custom-made mouthguard (occlusal thickness of 3-mm; 3MG) and no mouthguard (NoMG). Eleven male amateur contact team sports players completed three running incremental tests on different days. Each test was performed with either $3 \mathrm{MG}, 5 \mathrm{MG}$, or without a mouthguard. There were no significant differences in peak velocity during the incremental tests among conditions $\left(14.9 \pm 0.6,14.9 \pm 0.7\right.$, and $14.7 \pm 0.9 \mathrm{~km} \cdot \mathrm{h}^{-1}$ for NoMG, 3MG, and 5MG, respectively). Furthermore, no differences were found in the peaks of pulmonary oxygen uptake, minute ventilation, and respiratory frequency, as well as second ventilatory threshold. RPE was higher when wearing $5 \mathrm{MG}$ than when running without a mouthguard only at the $12.5 \mathrm{~km} \cdot \mathrm{h}^{-1}$ stage $(\mathrm{p}=.03)$. These data indicate that wearing custom-made mouthguards with occlusal thicknesses between 3- and 5-mm does not alter ventilatory parameters at the end of an incremental test. Thus, custom-made mouthguards with an occlusal thickness of 5-mm should be preferred owing to their greater protection capacity.
\end{abstract}

Key words: running, orofacial injury, $\mathrm{VO}_{2}$, athlete, oral airflow, exercise

\section{Introduction}

Participation in many sport activities puts athletes at risk of orofacial trauma, especially in team and contact sports that belong to the category of sports with a high risk of dental trauma (Knapik, et al., 2007; Kumamoto \& Maeda, 2004). Intra-oral mouthguards are used to prevent orofacial trauma, temporomandibular joint damage, soft tissue lacerations, and mandible fractures (Knapik, et al., 2007). These devices are designed to absorb the energy associated with a shock to the mouth, thus attenuating or preventing injuries (Bemelmanns \& Pfeiffer, 2001). In a recent meta-analysis, Emery et al. (2017) reported the reduced risk of concussion by $19 \%$ in studies examining the preventive effect of wearing a mouthguard. In addition, Knapik et al. (2007) indicated that when a mouthguard was not used, the overall risk of an orofacial injury was 1.6 to 1.9 times higher than when a mouthguard was worn. Thus, the use of mouthguard should be encouraged in sports where there is a risk of orofacial trauma (Knapik, et al., 2007).

There are three main categories of mouthguards: stock, self-adapted or "boil and bite", and custommade. Stock mouthguards are ready-made and inexpensive but not individually adapted (Delaney \& Montgomery, 2005). Self-adapted or "boil and bite" mouthguards are heated in water and then molded to the upper teeth by the athlete (Delaney \& Montgomery, 2005). Both these types do not have a good 
fit and are uncomfortable. By contrast, custommade mouthguards are fabricated personally by a dentist using a model of the athlete's mouth and a hard material such as ethylene vinyl acetate (EVA). Consequently, custom-made mouthguards have a better fit than stock and self-adapted mouthguards (Bemelmanns \& Pfeiffer, 2001; Drum, Swisher, Buchanan, \& Donath, 2016) and are believed to offer the best protection against orofacial injury due to their better retention in the mouth (Drum, et al., 2016; Kececi, Cetin, Eroglu, \& Baydar, 2005; Padilla, 2000). Thus, custom-made mouthguards are most recommended by dentists and preferred by sports practitioners because of a superior protection, fit, and comfort (Duarte-Pereira, et al., 2008).

An important factor about mouthguard protection ability is the occlusal thickness (Bemelmanns \& Pfeiffer, 2001; Knapik, et al., 2007; Verissimo, et al., 2016; Westerman, Stringfellow, \& Eccleston, $1995,2002)$. Westerman et al. $(1995,2002)$ showed that thicker mouthguards transmit less force to the mouth owing to their higher energy absorption capacity. However, recently it has been reported that wearing mouthguards with the occlusal thickness between 2- and 6-mm results in different extents of teeth strain (Verissimo, et al., 2016). While wearing mouthguards with the 2-mm occlusal thickness resulted in a higher teeth strain, a lower teeth strain was obtained with mouthguards with the occlusal thickness of 5-mm (Verissimo, et al., 2016). Hence, mouthguards with occlusal thickness between 3- and 5-mm could be recommended for use in sport activities (Verissimo, et al., 2016; Westerman, et al., 1995, 2002). Although thicker mouthguards provide better mouth protection, it is believed that they interfere with ventilation during exercise owing to a higher resistance to or restriction of oral airflow (Amis, Di Somma, Bacha, \& Wheatley, 2000; Bourdin, et al., 2006; Francis \& Brasher, 1991), cause discomfort (Westerman, et al., 2002), increase the tension between the lips and the cheeks (Verissimo, et al., 2016), have poor acceptance (Delaney \& Montgomery, 2005; Verissimo, et al., 2016; Westerman, et al., 2002), and reduce the ability to breath and communicate (Bailey, et al., 2015; Delaney \& Montgomery, 2005; Drum, et al., 2016; Westerman, et al., 2002); all these factors could affect the rate of perceived exertion (RPE) and performance during exercise.

Several investigations have been conducted to evaluate the effects of wearing different kinds of mouthguards during exercise (Allen, et al., 2018; Amis, et al., 2000; Arent, McKenna, \& Golem, 2010; Bailey, et al., 2015; Bourdin, et al., 2006; DuartePereira, et al., 2008; Dunn-Lewis, et al., 2012; Francis \& Brasher, 1991; Gebauer, Williamson, Wallman, \& Dawson, 2011; Rapisura, Coburn, Brown, \& Kersey, 2010). In general, anaerobic performance during tests such as countermove- ment jumping (Cetin, Kececi, Erdogan, \& Baydar, 2009; Ebben, Flanagan, \& Jensen, 2008), running sprint (Cetin, et al., 2009; Dunn-Lewis, et al., 2012), vertical jump (Arent, et al., 2010), and the Wingate Anaerobic Test (Arent, et al., 2010; Cetin, et al., 2009; Morales, Busca, Solana-Tramunt, \& Miro, 2015 ) is maintained or improved when wearing mouthguards. The results are inconclusive when it comes to aerobic exercise when wearing stock or self-adapted mouthguards (Bailey, et al., 2015; Delaney \& Montgomery, 2005; Francis \& Brasher, 1991; Garner \& McDivitt, 2009; Rapisura, et al., 2010). The studies that investigated the effects of custom-made mouthguards on aerobic parameters tended to conclude that the performance was neither improved nor worsened (Bourdin, et al., 2006; Gebauer, et al., 2011; Kececi, et al., 2005; Piero, et al., 2015; von Arx, Flury, Tschan, Buergin, \& Geiser, 2008). Even though previous studies have measured ventilatory parameters and performance indices in different sports, there are no data on the impact of occlusal thickness of custom-made mouthguards. In addition, the influence of occlusal thickness on the ventilatory parameters and exercise responses has not yet been considered or investigated. Therefore, it is currently unknown if thicker custommade mouthguards, which provide better protection to the mouth and could restrict the oral airflow (Amis, et al., 2000), have any influence on ventilatory parameters, RPE and peak velocity during the incremental test $\left(\mathrm{V}_{\mathrm{PEAK}}\right)$. Thus, the purpose of this study was to analyze the effects of occlusal thickness of custom-made mouthguards (3- and 5-mm of occlusal thickness: $3 \mathrm{MG}$ and $5 \mathrm{MG}$, respectively) on exercise capacity, cardiopulmonary, and perceptual responses to the incremental running test. It was hypothesized that thicker custom-made mouthguards could affect ventilatory parameters and RPE because of their higher resistance to oral airflow and discomfort, thus impairing the exercise capacity.

\section{Methods}

To determine whether occlusal thickness impacted $V_{\text {PEAK }}$, RPE, and ventilatory parameters, a repeated-measures study design protocol was utilized. While a wide variety of mouthguards can be chosen to investigate the effects on performance and physiological variables, we sought to determine the impact of custom-made mouthguards (owing to their better fit and protection) with different occlusal thicknesses. The independent variable was mouthguard presence and its thickness, whereas the dependent variables included $\mathrm{V}_{\mathrm{PEAK}}, \mathrm{RPE}$, and ventilatory parameters.

\section{Subjects}

Eleven male amateur players (age 19.3 \pm 3.1 years, body mass $76.5 \pm 5.8 \mathrm{~kg}$, body height 
$181.7 \pm 10.2 \mathrm{~cm}$ ) of contact team sports (i.e., basketball, rugby, or soccer) participated in this study. All subjects trained on a regular basis 4-6 times a week. The study was performed according to the Declaration of Helsinki, and the protocol was approved by the Institutional Ethics Committee for Research on Human Subjects. Before participation in the study, the subjects and/or their parents or guardians were informed of all test procedures, and they provided voluntary written informed consent to participate. Subjects were instructed to avoid ingesting caffeine or alcohol and were advised to maintain a regular diet and avoid strenuous exercise 24 hours preceding a test session. All participants were unaware of the purpose of the study. None of the subjects had a temporomandibular joint disorder, wore a removable dental appliance, or had fixed orthodontic appliances. In addition, none of the subjects had used a mouthguard previously.

\section{Procedures}

The subjects reported to the laboratory on four different days. During the first visit, two custommade mouthguards (i.e., $3 \mathrm{MG}$ and $5 \mathrm{MG}$ ) were individually molded. During the three subsequent visits, subjects performed incremental tests until exhaustion on a motorized treadmill (Millenium Super ATL, Inbramed, Porto Alegre, Brazil) while wearing one of the two custom-made mouthguards with different occlusal thicknesses, or wearing no mouthguard. During each test, the subjects breathed through a silicone face mask, which covered the mouth and nose, connected to an automated opencircuit gas analysis system (Quark CPET; Cosmed Srl, Rome, Italy). The respiratory gas exchange was measured breath-by-breath. Before each test, gas analyzers were calibrated using ambient air and gases containing $16 \%$ oxygen and $5 \%$ carbon dioxide. The turbine flow meter used for the determination of minute ventilation was calibrated with a 3-L calibration syringe (Cosmed Srl). All tests were performed at the same time of day $( \pm 2 \mathrm{~h})$. The protocols were conducted in a random order and completed within 3 weeks (one test per week).

Mouthguards production. Upper and lower jaw impressions were taken with standard trays using an alginate impression material (Jeltrate Plus, Dentsply, York, USA), disinfected using 1\% sodium hypochlorite, and poured with plaster (type III plaster Dent-Mix, Asfer, São Caetano do Sul, Brazil) to produce the working cast. The mouthguards were made for the upper arch, and moldings in the lower jaw were needed for better occlusal stability in the process. EVA (Dentsply, York, USA) sheets with thicknesses of 4- and 2-mm were used to fabricate the mouthguards. The sheets were placed in a thermal forming machine (Vacuum Laminator, BioArt, São Carlos, Brazil). They were heated for approximately two minutes and then formed on the models. The sheets were cooled down at room temperature in order to avoid deformation during removal from the model. For the mouthguards with the occlusal thickness of 3-mm we used an EVA sheet of 4-mm, which was outworn until the thickness reached 3-mm as determined with a thickness measuring device (Compass, Golgran, São Caetano do Sul, Brazil). For the mouthguards with the occlusal thickness of 5-mm, two EVA plates, one with a thickness of 4-mm and another of 2-mm, were placed in the thermoforming machine. The resulting sheet was outworn to reach a thickness of 5-mm as determined with the thickness measuring device. The plates were thicker than desired for manufacturing the mouthguard because the thickness of an EVA sheet decreases from its original value when heated (Padilla, 2000). The design of the mouthguards took in account the geometrical constraints: 1) up to the gingival mucosa and around the anatomic structures on the labial side; 2) about $10-\mathrm{mm}$ above the gingival margin in the palatal region; 3) enclosing the maxillary teeth to the distal surface of the first molars; 4) occlusal thicknesses of 3- and 5-mm. The fit, stability, and retention of mouthguards were checked when worn by the athletes. Adjustments were performed at that time, if needed. The athletes were instructed to wear the mouthguards interchangeably during their weekly workouts to improve the fit and prevent discomfort during the tests. The athletes were instructed to wear one mouthguard during one training day and the other mouthguard during the next workout session. No instructions were given to clench or not to clench the mouthguards during the tests (Ebben, et al., 2008).

Exercise protocol. Before starting the incremental tests, the subjects put a mouthguard (3MG or $5 \mathrm{MG}$ ) in the mouth and remained seated for three minutes for baseline measurements. The treadmill gradient was set to $1 \%$ (Jones \& Doust, 1996), and the initial velocity was set to $8 \mathrm{~km} \cdot \mathrm{h}^{-1}$ and increased without interruptions by $0.5 \mathrm{~km} \cdot \mathrm{h}^{-1}$ every minute. The time to exhaustion measurements started when the participant released the support rails and started running on the treadmill belt. $V_{\text {PEAK }}$ was calculated as the velocity of the last stage after its full completion, plus, if necessary, the fraction of time spent at the stage at which exhaustion occurred multiplied by $0.5 \mathrm{~km} \cdot \mathrm{h}^{-1}$. All tests were performed until exhaustion and were terminated when the subject could not maintain the stipulated velocity. At the end of each stage, RPE was recorded using the Borg 1-10 category scale (Borg, 1982).

Gas exchange measurements. The breathby-breath pulmonary oxygen uptake $\left(\mathrm{VO}_{2}\right)$ data from each test were initially examined to exclude occasional errant breath values, i.e., values more than three standard deviations from the local mean (i.e., five-point rolling mean). Thereafter, $\mathrm{VO}_{2}$ data 
were reduced to 15 -second average values, and the highest 15 -second $\mathrm{VO}_{2}$ value reached was considered $\mathrm{VO}_{2}$ peak. The last 15 -second $\mathrm{VO}_{2}$ value while the subject remained seated was considered $\mathrm{VO}_{2}$ rest. An identical procedure was employed to determine the rest and peak minute ventilation (VE) and respiratory frequency (Rf). The second ventilatory threshold $\left(\mathrm{VT}_{2}\right)$ was determined breath-by-breath based on increases in both ventilatory equivalent for oxygen and carbon dioxide and a decrease in end-tidal partial pressure of carbon dioxide (Davis, 1985). The second ventilatory threshold was determined by two independent researchers, with the third investigator resolving disagreements.

\section{Statistical analyses}

The results are expressed as mean \pm standard deviation. Comparisons were performed with the mixed linear modeling procedure of IBM SPSS Statistics (version 19.0, I IBM Corporation, New York, USA) to quantify the changes in ventilatory parameters, RPE, and $\mathrm{V}_{\mathrm{PEAK}}$ while wearing $3 \mathrm{MG}$, $5 \mathrm{MG}$, or no mouthguard (NoMG). The subject term was included in the model as a random effect, the condition (3MG, 5MG, or NoMG) was included as a fixed effect, and order of condition was included as an additional fixed effect to account for continuing familiarization or other order effects. For RPE responses, moment was also included as a fixed factor. Where a significant interaction effect was obtained, a post-hoc analysis was performed to identify the source of differences by using Bonferroni-adjusted $t$-tests. In all cases, the level of statistical significance was set at $\mathrm{p}<.05$.

\section{Results}

There were no differences in $\mathrm{VO}_{2}, \mathrm{VE}, \mathrm{Rf}$, and RPE between the conditions (3MG, 5MG, NoMG) during rest and in peak values during the incremental tests $(\mathrm{p}>.05$; Table 1 and Figure 1). Furthermore, there were no significant differences

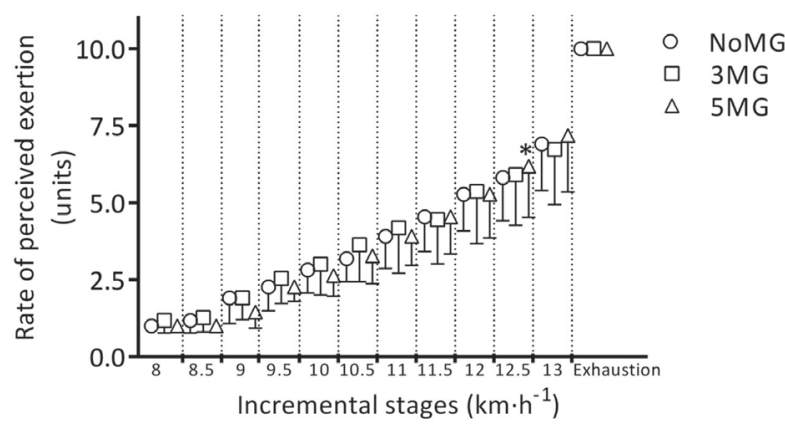

Figure 1. The rate of perceived exertion during the incremental test stages. NoMG: no mouthguard; $3 M G$ : custom-made mouthguard with an occlusal thickness of 3-mm; 5MG: custom-made mouthguard with an occlusal thickness of 5-mm. ${ }^{*} p=.03$, the rate of perceived exertion was higher with $5 M G$ than NoMG at $12.5 \mathrm{~km} \cdot \mathrm{h}^{-1}$.

in $\mathrm{VT}_{2}$ and $\mathrm{V}_{\mathrm{PEAK}}$ under different conditions $(\mathrm{p}<.05$; Table 1).

As all the athletes completed at least the 13 $\mathrm{km} \cdot \mathrm{h}^{-1}$ stage, RPE during the incremental tests was analyzed for velocities from $8 \mathrm{~km} \cdot \mathrm{h}^{-1}$ to $13 \mathrm{~km} \cdot \mathrm{h}^{-1}$. Similar to time effect $(\mathrm{p}<.001)$, the mixed modeling revealed a significant effect of mouthguard worn on RPE ( $\mathrm{p}=.04)$, and post-hoc analysis showed that RPE was higher for $5 \mathrm{MG}$ at $12.5 \mathrm{~km} \cdot \mathrm{h}^{-1}$ than for NoMG ( $p=.03$; Figure 1).

\section{Discussion and conclusions}

To our knowledge, this is the first study evaluating ventilatory parameters of athletes wearing mouthguards with different occlusal thicknesses. One of the main results of this study is that cardiopulmonary and exercise capacity responses were unaffected by a thicker mouthguard, rejecting, in part, our hypothesis. Furthermore, the RPE was higher only at $12.5 \mathrm{~km} \cdot \mathrm{h}^{-1}$ when wearing $5 \mathrm{MG}$ compared to NoMG.

Similar to the results of previous investigations, the present data suggest that the use of a mouthguard has no negative effects on athletes' $\mathrm{VO}_{2}$ peak.

Table 1. Ventilatory parameters, rate of perceived exertion, and peak velocity recorded during incremental tests with 3-and 5-mm mouthguards and without a mouthguard

\begin{tabular}{lcccc}
\hline Variable & NoMG & 3MG & $5 M G$ & $\begin{array}{c}\text { Main effects for condition } \\
(\mathrm{p} \text { value })\end{array}$ \\
\hline $\mathrm{VO}_{2}$ rest $\left(\mathrm{ml} \cdot \mathrm{min}^{-1}\right)$ & $373 \pm 69$ & $373 \pm 58$ & $364 \pm 100$ & .88 \\
$\mathrm{VO}_{2}$ peak $\left(\mathrm{ml} \cdot \mathrm{min}^{-1}\right)$ & $4561 \pm 582$ & $4453 \pm 529$ & $4423 \pm 525$ & .35 \\
VE rest $\left(\mid \cdot \mathrm{min}^{-1}\right)$ & $11 \pm 2$ & $11 \pm 2$ & $10 \pm 2$ & .72 \\
VE peak $\left(I \cdot \mathrm{min}^{-1}\right)$ & $156 \pm 19$ & $153 \pm 22$ & $151 \pm 25$ & .69 \\
Rf rest $\left(\mathrm{b} \cdot \mathrm{min}^{-1}\right)$ & $16 \pm 3$ & $17 \pm 4$ & $17 \pm 4$ & .74 \\
Rf peak $\left(\mathrm{b} \cdot \mathrm{min}^{-1}\right)$ & $66 \pm 13$ & $66 \pm 11$ & $65 \pm 10$ & .83 \\
$\mathrm{VT}_{2}\left(\mathrm{~km} \cdot \mathrm{h}^{-1}\right)$ & $11.4 \pm 0.8$ & $11.4 \pm 1.0$ & $11.3 \pm 0.9$ & .73 \\
$\mathrm{~V}_{\text {PEAK }}\left(\mathrm{km} \cdot \mathrm{h}^{-1}\right)$ & $14.9 \pm 0.6$ & $14.9 \pm 0.7$ & $14.7 \pm 0.9$ & .98 \\
\hline
\end{tabular}

Note. Data are presented as mean \pm standard deviation. NoMG: no mouthguard condition. 3MG: custom-made mouthguard with 3-mm of occlusal thicknesses. 5MG: custom-made mouthguard with 5-mm of occlusal thicknesses. $\mathrm{VO}_{2}$ : pulmonary oxygen uptake. $\mathrm{VE}$ : minute ventilation. $\mathrm{Rf}$ : respiratory frequency. $\mathrm{VT}_{2}$ : second ventilatory threshold. $\mathrm{V}_{\mathrm{PEAK}}$ : peak velocity. 
Piero et al. (2015) recently showed, in male amateur road cyclists, that neither the peak nor submaximal $\mathrm{VO}_{2}$ values of athletes were negatively influenced by a custom-made mouthguard compared to NoMG. In addition, Bourdin et al. (2006) observed that $\mathrm{VO}_{2}$ peak was similar when wearing a custom-made or self-adapted mouthguard to that when wearing no mouthguard. Thus, although thicker mouthguards (e.g., 5MG) could potentially represent a larger obstruction in the oral breathing route, this was not sufficient to reduce athletes' ventilation and affect $\mathrm{VO}_{2}$ peak. This is not surprising, since athletes wearing mouthguards are able to utilize compensatory mechanisms for insufficient oral airflow such as an increase in mouth opening when at high ventilatory rates (Amis, et al., 2000).

Similarly, this study demonstrated that athletes' VE peak was not affected by the use of mouthguards. In agreement, von Arx et al. (2008) reported that VE peak was not negatively affected by the custommade mouthguard use during maximal intensity exercise on a cycle ergometer. In contrast, when the commercial self-adapted or "boil and bite" mouthguards were used, VE peak was reduced compared to the value without a mouthguard (Bailey, et al., 2015). These discrepant results can be attributed to differences between the types of mouthguards (Caneppele, et al., 2017), with the mouthguards that were not made individually being less efficient than the custom-made mouthguards used in the present study. In addition, no changes in $\mathrm{VT}_{2}$ and $\mathrm{Rf}$ peak were found between the different conditions, as was reported previously (Bourdin, et al., 2006; Piero, et al., 2015; von Arx, et al., 2008), suggesting that no ventilatory limitations occurred during the incremental tests with $3 \mathrm{MG}$ and $5 \mathrm{MG}$.

Despite no changes in the athletes' cardiorespiratory variables, the RPE was higher when wearing a $5 \mathrm{MG}$ at the velocity of $12.5 \mathrm{~km} \cdot \mathrm{h}^{-1}$ than without a mouthguard during the incremental test. Recently, Bailey et al. (2015) observed that perceptions of comfort, ability to breath, and ability to communicate were negatively affected by wearing mouth- guards during incremental tests. Furthermore, Delaney and Montgomery (2005) in their study of mouthguard perception showed that athletes felt that a mouthguard hindered their breathing. A limitation of the present study is a lack of a questionnaire to assess how the athletes perceived the mouthguards in terms of comfort and ability to breath. On the other hand, it has been shown that the perception of discomfort decreases after a fourweek period of using a mouthguard regularly (von Arx, et al., 2008). Therefore, even if RPE may be altered during exercise, frequent use of mouthguards (e.g., during training) should be encouraged to reduce the discomfort during matches and competitions and to increase acceptance.

The present findings on $\mathrm{V}_{\mathrm{PEAK}}$ are corroborated by other studies evaluating the effects of wearing custom-made mouthguards during incremental tests, which found no reductions at peak power on a cycle ergometer (Bourdin, et al., 2006; von Arx, et al., 2008). However, this is the first assessment of the effects of mouthguard use in terms of $\mathrm{V}_{\text {PEAK }}$ on a treadmill. In summary, the present results demonstrate that wearing custom-made mouthguards with occlusal thicknesses between 3 - and 5-mm does not alter cardiopulmonary and performance responses of athletes to an incremental exercise test.

\section{Practical applications}

Since custom-made mouthguards do not show negative effects on exercise performance and physiological parameters, it is recommended that athletes use them during training and competitions to prevent or mitigate orofacial injuries arising from falls, bumps, and knocks. Furthermore, custommade mouthguards with an occlusal thickness of 5-mm should be preferred due to their greater protection capacity. However, 5-mm custom-made mouthguards may cause discomfort because of their thickness. To reduce this discomfort, athletes should wear these mouthguards as often as possible during training to achieve a better fit, comfort, and adaptability.

\section{References}

Allen, C.R., Fu, Y.C., Cazas-Moreno, V., Valliant, M.W., Gdovin, J.R., Williams, C.C., \& Garner, J. C. (2018). Effects of jaw clenching and jaw alignment mouthpiece use on force production during vertical jump and isometric clean pull. Journal of Strength and Conditioning Research, 32(1), 237-243. doi: 10.1519/JSC.0000000000002172

Amis, T., Di Somma, E., Bacha, F., \& Wheatley, J. (2000). Influence of intra-oral maxillary sports mouthguards on the airflow dynamics of oral breathing. Medicine and Science in Sports and Exercise, 32(2), 284-290. doi: 10.1097/00005768-200002000-00006

Arent, S.M., McKenna, J., \& Golem, D.L. (2010). Effects of a neuromuscular dentistry-designed mouthguard on muscular endurance and anaerobic power. Comparative Exercise Physiology, 7(2), 73-79. doi: 10.1017/S1755254010000231 
Bailey, S.P., Willauer, T.J., Balilionis, G., Wilson, L.E., Salley, J.T., Bailey, E.K., \& Strickland, T.L. (2015). Effects of an over-the-counter vented mouthguard on cardiorespiratory responses to exercise and physical agility. Journal of Strength and Conditioning Research, 29(3), 678-684. doi: 10.1519/JSC.0000000000000668

Bemelmanns, P., \& Pfeiffer, P. (2001). Shock absorption capacities of mouthguards in different types and thicknesses. International Journal of Sports Medicine, 22(2), 149-153. doi: 10.1055/s-2001-11342

Borg, G.A. (1982). Psychophysical bases of perceived exertion. Medicine and Science in Sports and Exercise, 14(5), 377-381. doi: 10.1249/00005768-198205000-00012

Bourdin, M., Brunet-Patru, I., Hager, P.E., Allard, Y., Hager, J.P., Lacour, J.R., \& Moyen, B. (2006). Influence of maxillary mouthguards on physiological parameters. Medicine and Science in Sports and Exercise, 38(8), 15001504. doi: 10.1249/01.mss.0000228952.44850.eb

Caneppele, T.M.F., Borges, A.B., Pereira, D.M., Fagundes, A.A., Fidalgo, T.K.S., \& Maia, L.C. (2017). Mouthguard use and cardiopulmonary capacity - A systematic review and meta-analysis. Sports Medicine International Open, 1(05), E172-E182. doi: 10.1055/s-0043-117599

Cetin, C., Kececi, A.D., Erdogan, A., \& Baydar, M.L. (2009). Influence of custom-made mouth guards on strength, speed and anaerobic performance of taekwondo athletes. Dental Traumatology, 25(3), 272-276. doi: 10.1111/j.16009657.2009.00780.x

Davis, J.A. (1985). Anaerobic threshold: Review of the concept and directions for future research. Medicine and Science in Sports and Exercise, 17(1), 6-21.

Delaney, J.S., \& Montgomery, D.L. (2005). Effect of noncustom bimolar mouthguards on peak ventilation in ice hockey players. Clinical Journal of Sport Medicine, 15(3), 154-157. doi: 10.1097/01.jsm.0000158256.85664.8d

Drum, S.N., Swisher, A.M., Buchanan, C.A., \& Donath, L. (2016). Effects of a custom bite-aligning mouthguard on performance in college football players. Journal of Strength and Conditioning Research, 30(5), 1409-1415. doi: 10.1519/JSC.0000000000001235

Duarte-Pereira, D.M., Del Rey-Santamaria, M., Javierre-Garces, C., Barbany-Cairo, J., Paredes-Garcia, J., ValmasedaCastellon, E., .. ., \& Gay-Escoda, C. (2008). Wearability and physiological effects of custom-fitted vs selfadapted mouthguards. Dental Traumatology, 24(4), 439-442. doi: 10.1111/j.1600-9657.2008.00595.x

Dunn-Lewis, C., Luk, H.Y., Comstock, B.A., Szivak, T.K., Hooper, D.R., Kupchak, B.R., ..., \& Kraemer, W.J. (2012). The effects of a customized over-the-counter mouth guard on neuromuscular force and power production in trained men and women. Journal of Strength and Conditioning Research, 26(4), 1085-1093. doi: 10.1519/ JSC.0b013e31824b4d5b

Ebben, W.P., Flanagan, E.P., \& Jensen, R.L. (2008). Jaw clenching results in concurrent activation potentiation during the countermovement jump. Journal of Strength and Conditioning Research, 22(6), 1850-1854. doi: 10.1519/ JSC.0b013e3181875117

Emery, C.A., Black, A.M., Kolstad, A., Martinez, G., Nettel-Aguirre, A., Engebretsen, L., .. ., \& Schneider, K. (2017). What strategies can be used to effectively reduce the risk of concussion in sport? A systematic review. British Journal of Sports Medicine, 51(12), 978-984. doi: 10.1136/bjsports-2016-097452

Francis, K.T., \& Brasher, J. (1991). Physiological effects of wearing mouthguards. British Journal of Sports Medicine, 25(4), 227-231. doi: 10.1136/bjsm.25.4.227

Garner, D.P., \& McDivitt, E. (2009). Effects of mouthpiece use on airway openings and lactate levels in healthy college males. Compendium of Continuing Education in Dentistry, 30(Spec. No. 2), 9-13.

Gebauer, D.P., Williamson, R.A., Wallman, K.E., \& Dawson, B.T. (2011). The effect of mouthguard design on respiratory function in athletes. Clinical Journal of Sport Medicine, 21(2), 95-100. doi: 10.1097/JSM.0b013e31820428b0

Jones, A.M., \& Doust, J.H. (1996). A 1\% treadmill grade most accurately reflects the energetic cost of outdoor running. Journal of Sports Sciences, 14(4), 321-327. doi: 10.1080/02640419608727717

Kececi, A.D., Cetin, C., Eroglu, E., \& Baydar, M.L. (2005). Do custom-made mouth guards have negative effects on aerobic performance capacity of athletes? Dental Traumatology, 21(5), 276-280. doi: 10.1111/j.16009657.2005.00354.x

Knapik, J.J., Marshall, S.W., Lee, R.B., Darakjy, S.S., Jones, S.B., Mitchener, T.A., ..., \& Jones, B.H. (2007). Mouthguards in sport activities: History, physical properties and injury prevention effectiveness. Sports Medicine, 37(2), 117-144. doi: 10.2165/00007256-200737020-00003

Kumamoto, D.P., \& Maeda, Y. (2004). A literature review of sports-related orofacial trauma. General Dentistry, 52(3), 270-280; quiz 281.

Morales, J., Busca, B., Solana-Tramunt, M., \& Miro, A. (2015). Acute effects of jaw clenching using a customized mouthguard on anaerobic ability and ventilatory flows. Human Movement Science, 44, 270-276. doi: 10.1016/j. humov.2015.09.008

Padilla, R. (2000). Overcoming objections: Providing professionally made custom mouthguards. Dentistry Today, 19(9), 84-86, 88-89.

Piero, M., Simone, U., Jonathan, M., Maria, S., Giulio, G., Francesco, T., . ., \& Giovanni, G. (2015). Influence of a custom-made maxillary mouthguard on gas exchange parameters during incremental exercise in amateur road cyclists. Journal of Strength and Conditioning Research, 29(3), 672-677. doi: 10.1519/JSC.0000000000000695 
Rapisura, K.P., Coburn, J.W., Brown, L.E., \& Kersey, R. D. (2010). Physiological variables and mouthguard use in women during exercise. Journal of Strength and Conditioning Research, 24(5), 1263-1268. doi: 10.1519/ JSC.0b013e3181d1572d

Verissimo, C., Costa, P.V., Santos-Filho, P.C., Tantbirojn, D., Versluis, A., \& Soares, C.J. (2016). Custom-Fitted EVA Mouthguards: What is the ideal thickness? A dynamic finite element impact study. Dental Traumatology, 32(2), 95-102. doi: 10.1111/edt.12210

von Arx, T., Flury, R., Tschan, J., Buergin, W., \& Geiser, T. (2008). Exercise capacity in athletes with mouthguards. International Journal of Sports Medicine, 29(5), 435-438. doi: 10.1055/s-2007-965341

Westerman, B., Stringfellow, P.M., \& Eccleston, J.A. (1995). Forces transmitted through EVA mouthguard materials of different types and thickness. Australian Dental Journal, 40(6), 389-391. doi: 10.1111/j.1834-7819.1995.tb04838.x

Westerman, B., Stringfellow, P.M., \& Eccleston, J.A. (2002). EVA mouthguards: How thick should they be? Dental Traumatology, 18(1), 24-27. doi: 10.1034/j.1600-9657.2002.180103.x

Submitted: October 6, 2017

Accepted: September 5, 2018

Published Online First: November 14, 2019

Correspondence to:

João Antônio Gesser Raimundo, M.Sc.

Laboratório de Pesquisas em Desempenho Humano -

CEFID/UDESC

Rua Pascoal Simone, 358, Coqueiros - Florianópolis SC - Brazil

Phone: +55483664.8641

Fax: +55 483664.8607

E-mail: joaoagrgesser@hotmail.com

\section{Acknowledgments}

We would like to express our gratitude to Fernando Klitzke Borszcz for his technical assistance. This study was supported by the Coordination for the Improvement of Higher Level -or Education- Personnel (CAPES) and Santa Catarina State Research Foundation (FAPESC). 